\title{
Neuronal guidance molecule netrin-1 attenuates inflammatory cell trafficking during acute experimental colitis
}

\author{
Carol M Aherne, ${ }^{1}$ Colm B Collins, ${ }^{2}$ Joanne C Masterson, ${ }^{2,3}$ Marco Tizzano, ${ }^{4}$ \\ Theresa A Boyle, ${ }^{5}$ Joseph A Westrich, ${ }^{1}$ Jason A Parnes, ${ }^{4}$ Glenn T Furuta, ${ }^{2,3}$ \\ Jesús Rivera-Nieves, ${ }^{2}$ Holger K Eltzschig ${ }^{1}$
}

- Additional materials are published online only. To view these files please visit the journal online (http://gut.bmj. com/content/61/5.toc).

${ }^{1}$ Mucosal Inflammation Program, Department of Anesthesiology, University of Colorado Denver, Aurora, Colorado, USA

${ }^{2}$ Mucosal Inflammation Program, Department of Medicine, University of Colorado Denver, Aurora, Colorado, USA ${ }^{3}$ Gastrointestinal Eosinophilic Diseases Program, Section of Pediatric Gastroenterology, Hepatology and Nutrition, Digestive Health Institute, Children's Hospital Colorado Aurora, Colorado, USA

${ }^{4}$ Department of Cell and Developmental Biology, Rocky Mountain Taste and Smell Centre, University of Colorado, Denver, Aurora, Colorado, USA ${ }^{5}$ Department of Pathology, University of Colorado, Denver, Aurora, Colorado, USA

\section{Correspondence to}

Dr Holger K Eltzschig, Mucosal Inflammation Program, Department of Anesthesiology, University of Colorado Denver, 12700 E 19th Avenue, Mailstop B112, Research Complex 2 Room 7124, Aurora, CO 80045, USA; holger.eltzschig@ ucdenver.edu

Original data related to this publication are available upon request.

Revised 15 June 2011 Accepted 20 June 2011 Published Online First

3 August 2011

\section{UNLOCKA1}

This paper is freely available online under the BMJ Journals unlocked scheme, see http:// gut.bmi.com/site/about/ unlocked.xhtml

\section{ABSTRACT \\ Background Inflammatory bowel diseases,}

encompassing Crohn's disease and ulcerative colitis, are characterised by persistent leucocyte tissue infiltration leading to perpetuation of an inappropriate inflammatory cascade. The neuronal guidance molecule netrin-1 has recently been implicated in the orchestration of leucocyte trafficking during acute inflammation. We therefore hypothesised that netrin-1 could modulate leucocyte infiltration and disease activity in a model of inflammatory bowel disease.

Design DSS-colitis was performed in mice with partial genetic netrin-1 deficiency (Ntn-1 ${ }^{+/-}$mice) or wild-type mice treated with exogenous netrin-1 via osmotic pump to examine the role of endogenous and therapeutically administered netrin-1. These studies were supported by in vitro models of transepithelial migration and intestinal epithelial barrier function.

Results Consistent with our hypothesis, we observed induction of netrin-1 during intestinal inflammation in vitro or in mice exposed to experimental colitis. Moreover, mice with partial netrin-1 deficiency demonstrated an exacerbated course of DSS-colitis compared to littermate controls, with enhanced weight loss and colonic shortening. Conversely, mice treated with exogenous mouse netrin-1 experienced attenuated disease severity. Importantly, permeability studies and quantitative assessment of apoptosis reveal that netrin-1 signalling events do not alter mucosal permeability or intestinal epithelial cell apoptosis. In vivo studies of leucocyte transmigration demonstrate suppression of neutrophil trafficking as a key function mediated by endogenous or exogenously administered netrin-1. Finally, genetic studies implicate the A2B adenosine receptor in netrin-1-mediated protection during DSS-colitis.

Conclusions The present study identifies a previously unrecognised role for netrin-1 in attenuating experimental colitis through limitation of neutrophil trafficking.

\section{INTRODUCTION}

Netrin-1 was originally identified as a diffusible factor released by neural floor plate cells in the developing spinal cord that regulates axonal outgrowth. ${ }^{1}$ Studies have expanded to peripheral organs where netrin-1 modulates cell development, migration and inflammatory end points to reduce tissue inflammation. ${ }^{2-10}$

\section{Significance of this study}

What is already known about this subject?

- As a neuronal guidance molecule, netrin-1 has been implicated in the developing brain

- Previous studies have found high expression levels of netrin- 1 in the intestine, including in patients with inflammatory bowel disease (IBD)

- Netrin-1 has been implicated in immune functions

What are the new findings?

- At present, the functional role of netrin-1 in IBD is unknown. As such, our studies provide, for the first time, evidence for a protective role of netrin1 signalling in an experimental model of IBD

- Mice with a deficiency of netrin-1 experience enhanced disease severity during experimental colitis

- Treatment with exogenous netrin-1 using osmotic pump delivery attenuates experimental colitis

- Netrin-1 does not alter epithelial permeability or apoptosis in vivo but directly limits neutrophil trafficking to attenuate disease

How might it impact on clinical practice in the foreseeable future?

- Continuous low-dose administration of netrin-1 as demonstrated here in an experimental model of colitis may be exploited as a therapeutic agent directed towards the suppression of neutrophil infiltration in patients with IBD

Inflammatory bowel diseases (IBDs), encapsulating Crohn's disease (CD) and ulcerative colitis (UC), are heterogeneous diseases affecting the gastrointestinal tract that occur at the cross section of genetics and environment. ${ }^{11} 12$ Disruption of the intestinal epithelial barrier and aberrant accumulation of leucocytes within the intestinal lamina propria (LP) leading to continued mucosal damage characterise $\mathrm{CD}$ and $\mathrm{UC}{ }^{13}$ While biologics offer more targeted treatment, the search for increased specificity in IBD therapeutics continues. ${ }^{14}$

The role of netrin-1 in IBD remains undefined. Previous reports demonstrate enhanced netrin-1 expression in the intestinal epithelium of patients with CD or UC. ${ }^{15}$ Hypoxia-dependent signalling 
induces intestinal mucosal netrin-1 expression, ${ }^{2}$ with recent reports demonstrating a tissue-protective role for the hypoxic response in IBD. ${ }^{16-19}$ This notion coupled with an inhibitory effect of netrin-1 on leucocyte transmigration ${ }^{2}{ }^{6}$ led us to hypothesise that netrin-1 may mediate tissue protection in IBD. We established that netrin-1 expression was enhanced within the colonic mucosa during murine colitis. Deficiency of netrin-1 resulted in exacerbated murine colitis accompanied by exaggerated neutrophil infiltration into the colonic LP. This was attenuated by recombinant netrin-1. Mechanistic studies reveal that netrin-1 mediates its effects through interaction with migrating leucocytes without directly modulating intestinal epithelial barrier function or epithelial cell apoptosis. Furthermore, we demonstrate a role for the purinergic signalling pathway in netrin-1-mediated protection. Our data demonstrate, for the first time, a protective role for netrin-1 in experimental colitis.

\section{MATERIALS AND METHODS Cell culture}

Caco-2, T84 and HMEC-1 cells were cultured as described. ${ }^{20-22}$ Time course studies were performed with recombinant human tumor necrosis factor (TNF) $\alpha$, interleukin (IL) $\beta$ and interferon (IFN) $\gamma$.

\section{DSS-colitis}

Netrin-1 heterozygous mice (Ntn-1${ }^{+/-}$mice), A2B adenosine receptor-deficient (Adora $2 \mathrm{~b}^{-1-}$ ) mice, matched littermate controls or C57BL/6 mice were used in DSS (dextran sulphate sodium) studies as described. ${ }^{23-25}$ Recombinant netrin-1 was delivered with subcutaneous osmotic pumps ( $1 \mu \mathrm{g} / \mathrm{mouse} / \mathrm{day})$. Anti-UNC5B antibody was administered intraperitoneally (800 $\mu \mathrm{g} / \mathrm{kg} /$ mouse).

\section{Flow cytometry}

Flow cytometric analysis of LP leucocytes with anti-mouse GR-1, SiglecF, Ly-6G, Ly-6C, CD11b, CD45, LIVE/DEAD® cell stain or isotype controls was performed as described. ${ }^{26}$

\section{Immunohistochemistry}

Paraffin-embedded tissues were incubated with anti-mouse netrin-1 followed by $\mathrm{DAB}$ (3,3-diaminobenzidine substrate) development and methyl green counterstain.

\section{Immunofluorescence}

Frozen tissues were incubated with anti-PGP9.5 or anti-CGRP, followed by fluorescent detection and DAPI (4',6-diamidino-2phenylindole) counterstain.

\section{Western blotting}

Tissue immunoblotting was performed as described. ${ }^{2}$

\section{Real-time reverse transcriptase polymerase chain reaction (RT-PCR)}

Messenger RNA (mRNA) analysis was performed as described. ${ }^{2}$

\section{In vivo permeability}

FITC-labelled (fluorescein isothiocyanate) dextran permeability was performed as described. ${ }^{19}$

\section{TUNEL assay}

TUNEL (deoxynucleotidyl transferase-mediated deoxyuridine triphosphate) assay using paraffin-embedded tissues was performed as per manufacturer's instructions.

\section{In vitro permeability}

Permeability studies in Caco-2 cells treated with DSS or cytokines were performed as described. ${ }^{20}$

\section{In vitro transmigration}

Polymorphonuclear leucocytes (PMN) transmigration was performed as described. ${ }^{2}$

\section{Statistical analysis}

Statistical comparisons were made with GraphPad Prism Analysis software using analysis of variance (ANOVA) or Student $t$ test where appropriate and are expressed at mean \pm SEM.

Further details can be found in the supplementary material.

\section{RESULTS}

Induction of netrin-1 expression during intestinal inflammation Previous study suggests that inflammation regulates netrin-1 expression. ${ }^{27}$ To examine the effects of inflammation on intestinal expression of netrin-1, we assessed netrin- 1 mRNA levels in human intestinal epithelial cell lines (T84 and Caco-2; figure 1A, $\mathrm{B}$, respectively) and a human microvascular endothelial cell line (HMEC-1; figure 1C) following $2 \mathrm{~h}$ and $6 \mathrm{~h}$ stimulation with a combination of mediators closely associated with IBD pathogenesis (cytomix; TNF $\alpha$, IL-1 $\beta$ and IFN $\gamma$ ). Netrin-1 mRNA expression significantly increased in all cell lines following $6 \mathrm{~h}$ treatment. To examine this response and identify the cellular source of netrin-1 in vivo, netrin-1 expression was analysed in a DSS-colitis time course (figure 1D-G). Immunohistochemical analysis demonstrated robust expression of netrin-1 in colonic epithelial cells and in myenteric neural units (figure 1D; arrows identify staining of relevant cells). PCR analysis of HMEC-1 cells revealed induction of netrin-1 mRNA upon inflammatory stimulation (figure 1C); however, immunohistochemistry failed to show netrin-1 expression in colonic endothelial cells during DSS. Netrin-1 expression remained specifically localised to the intestinal epithelium and the myenteric neural units of the colon throughout the DSS time course, with the appearance of increasing expression over time. mRNA (figure 1E) and western blotting (figure $1 \mathrm{~F}, \mathrm{G}$ ) confirmed a quantifiable increase in netrin1 expression in the whole colon (figure $1 \mathrm{E}, \mathrm{F}$ ) and in the mucosal lining layer (figure 1G) following 7 days of DSS. These observations indicate induction of netrin-1 expression during experimental colitis with significant expression by the epithelium.

\section{Mice with partial netrin-1 deficiency demonstrate reduced netrin-1 expression and normal enteric neuronal anatomy in experimental colitis}

Netrin-1-deficient mice do not survive beyond postnatal day 3 due to improper axonal development. ${ }^{28}$ Mice with partial netrin1 deficiency $\left(\mathrm{Ntn-1^{+/- }}\right.$ ) develop normally compared to wild-type controls $\left(\mathrm{Ntn-1}{ }^{+/+}\right) .{ }^{28}$ Having observed netrin-1 expression in the colon following experimental colitis, we assessed netrin-1 expression in $N t n-1^{+/-}$mice following DSS. Netrin-1 expression in $N t n-1^{+/-}$mice remained below $N t n-1^{+/+}$at baseline and was similarly reduced following DSS (figure $2 \mathrm{~A}-\mathrm{C}$ ). Therefore, we conclude that during DSS-colitis, endogenous netrin-1 levels are dramatically diminished in $N t n-1^{+/-}$mice.

Initial findings demonstrated netrin-1 expression in colonic myenteric neural units (figure 1D); therefore, we compared the colonic neuronal anatomy of $N t n-1^{+/-}$and $N t n-1^{+/+}$mice at baseline and during DSS. Staining for peripheral nerve fibres (anti-PGP9.5) revealed no appreciable difference in the neuronal anatomy of the colon of $\mathrm{Ntn-1} 1^{+/-}$mice compared to $\mathrm{Ntn-1^{+/+ }}$ prior to or during DSS-colitis (figure 2D). Additionally, no difference in pain nerve fibres was observed between groups (anti-CGRP; data not shown). We therefore conclude that mice with netrin-1 insufficiency do not demonstrate altered neuronal patterning during DSS. 
Figure 1 Netrin-1 expression in response to inflammation. T84 intestinal epithelial cells (A), Caco-2 intestinal epithelial cells (B) or HMEC-1 microvascular endothelial cells (C) were exposed to a combination of tumor necrosis factor (TNF) $\alpha$, interleukin (IL) $1 \beta$ and interferon (IFN) $\gamma$ (cytomix; all at $10 \mathrm{ng} / \mathrm{ml})$ or vehicle (0.1\% bovine serum albumin (BSA)/phosphatebuffered saline (PBS)) for $2 \mathrm{~h}$ or $6 \mathrm{~h}$ followed by total RNA isolation and analysis of netrin-1 transcript levels (NTN-1). NTN-1 expression was calculated relative to $\beta$-actin and expressed as fold change relative to vehicle treatment \pm SEM for each timepoint. Results are derived from independent experiments at each individual time point, performed in triplicate. (D) Gender-, age- and weightmatched $\mathrm{C} 57 \mathrm{BL} / 6$ mice exposed to water or DSS $(4.5 \%)$ over a timecourse of 7 days were sacrificed at 2-day intervals post DSS administration (days $0,1,3,5$ and 7), and whole colon was harvested by blunt dissection. Paraffinembedded sections of whole colon were deparaffinised and rehydrated for immunohistochemical staining with a chicken anti-netrin-1 specific antibody (1:100) followed by DAB development (brown staining) and counterstaining with methyl green (green staining). Primary antibody was omitted as negative control (control).

Representative images were acquired at 10X using a Nikon Eclipse Ti-S microscope, DS-Fi1 1.0X camera. Bar represents $100 \mu \mathrm{m}$. (E) Total RNA was isolated from whole colonic tissue isolated at different time intervals of DSS administration as described (D), and netrin-1 transcript levels (Ntn-1) were determined by real-time reverse transcriptase polymerase chain reaction (RT-PCR). Ntn-1 expression was calculated relative to $\beta$-actin and expressed as fold change relative to water-exposed mice \pm SEM. (F) Whole
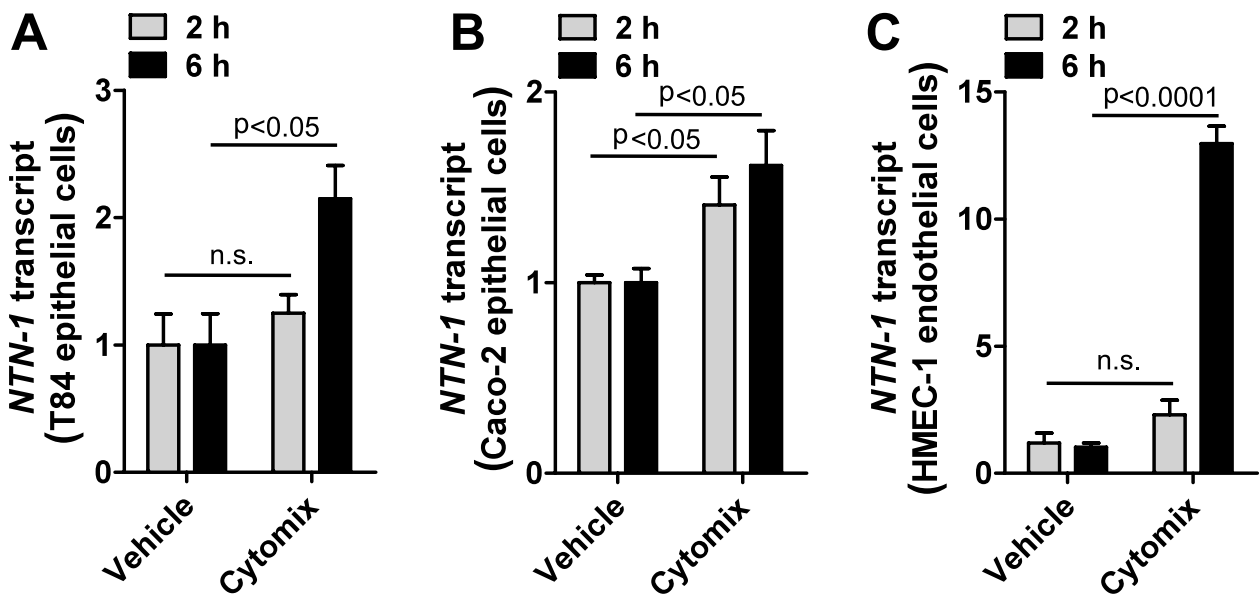

D
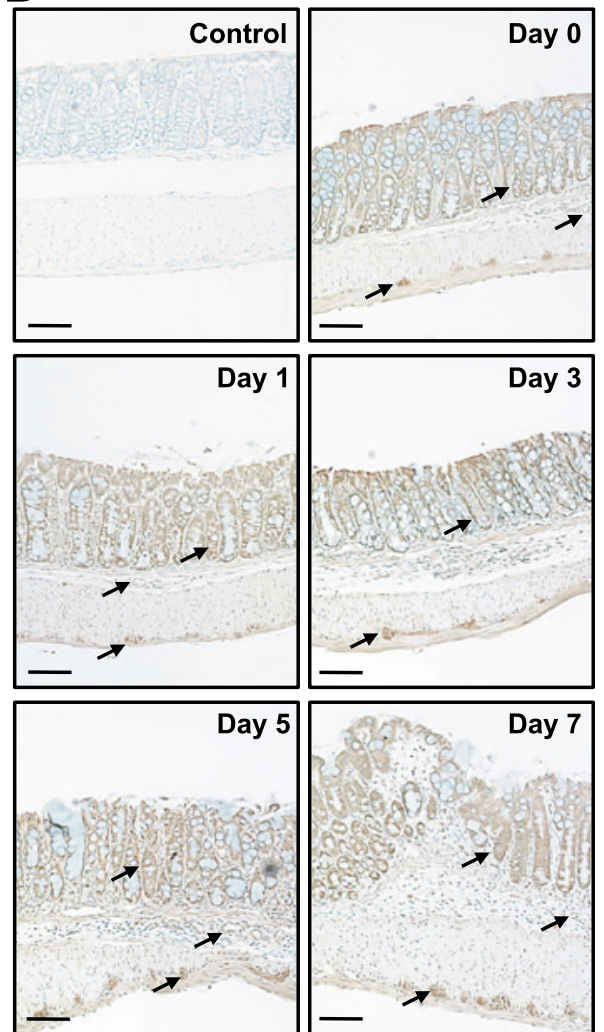

E

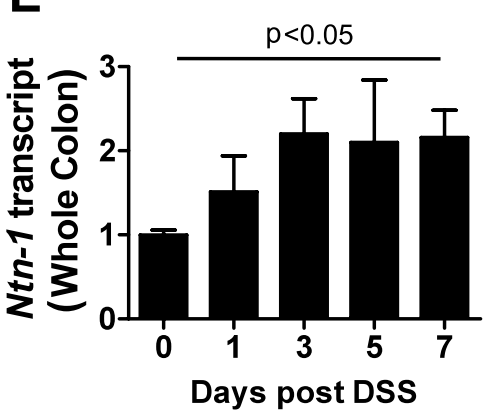

$\mathbf{F}$ determined by sodium dodecyl sulfate polyacrylamide gel electrophoresis (SDS-PAGE) with $\beta$-actin as loading control. (G) Colonic mucosal scrapings were harvested post DSS (described in D). Netrin-1 (Ntn-1) expression in the mucosal layer at 7 days post DSS was determined by SDS-PAGE with $\beta$ actin as loading control. In vivo DSS time course data represent five mice per time point, and western blot data are representative of greater than three independent experiments with six to eight mice per group.

\section{Partial netrin-1 deficiency exacerbates disease activity in a model of experimental colitis}

Recent study indicates that mice with partial genetic defi-

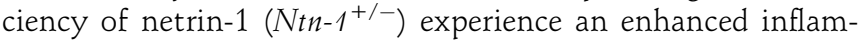
matory response in models of acute inflammation, ${ }^{2} 89$ thus prompting our investigation of these mice in experimental colitis. Consistent with our hypothesis, we observed that $N t n-1^{+/-}$mice experienced increased disease severity compared to wild-type controls $\left(\mathrm{Ntn-1^{+/+ }}\right)$ as measured by weight loss, disease activity index, colonic shortening and histologic damage (figure $3 \mathrm{~A}-\mathrm{E}$, respectively). Thus, we conclude that partial deficiency of netrin-1 is detrimental to the development of DSS-colitis.

\section{Partial netrin-1 deficiency results in heightened colonic} inflammation in experimental colitis

Previous observations demonstrate that netrin- 1 can inhibit leucocyte tissue infiltration, with studies demonstrating netrin-1 inhibition of neutrophil and monocyte recruitment in acute inflammation. ${ }^{2}{ }^{6-9}$ Inappropriate neutrophil accumulation within the LP is a key feature of early and active UC. ${ }^{29-31}$ DSScolitis is characterised by rapid neutrophil influx into the LP, closely modelling human disease..$^{32}$ Rapid onset of weight loss in the $\mathrm{Ntn-1}^{+/-}$mice prompted investigation of early and late disease activity. Flow cytometric analysis of leucocytes isolated from the whole colon following 3 and 7 days of DSS revealed no significant difference in dendritic cell $\left(\mathrm{CD} 11 \mathrm{c}^{+} \mathrm{MHCII}{ }^{\mathrm{Hi}}\right)$, 
Figure 2 Netrin-1 expression and enteric neuronal patterning in mice with partial netrin-1 deficiency following DSS-colitis. Age-, weight-, and sexmatched mice with partial netrin-1 deficiency (Ntn-1 $\left.{ }^{+/-}\right)$and their wildtype controls $\left(\mathrm{Ntn}-1^{+/+}\right)$were exposed to water or DSS (4.5\%) for 7 days, followed by sacrifice and harvesting of the whole colon by blunt dissection. (A) Total RNA was extracted, and Ntn-1 transcript levels determined by realtime reverse transcriptase polymerase chain reaction (RT-PCR). Ntn-1 expression was calculated relative to $\beta$-actin and expressed as fold change relative to water-exposed $\mathrm{Ntn}-\mathrm{1}^{+/+}$ mice \pm SEM. (B) Total protein was extracted, and Ntn-1 levels were determined by sodium dodecyl sulfate polyacrylamide gel electrophoresis (SDS-PAGE) with $\beta$-actin as the loading control. (C) Densitometric analysis of netrin-1 expression in mucosal scrapings as mean fold change relative to water-exposed $\mathrm{Ntn}-1^{+/+}$

mice \pm SEM. Results are representative of at least three independent experiments with six to eight mice per group. (D) Representative images of immunofluorescence staining performed on frozen sections from whole colon of mice with partial netrin1 deficiency $\left(\mathrm{Ntn}-1^{+/-}\right)$or wild-type controls

$\left(N t n-1^{+/+}\right)$exposed to water or DSS using an anti-PGP9.5 antibody (red) and counterstaining with DAPI (blue). Images were acquired with a 0lmaging Retiga-400RV at 10X. Bar represents $100 \mu \mathrm{m}$. Results represent analysis of mice at 3 and 7 days post DSS stained as a whole mount and in sequential sections ( $n=2$ mice per group).

natural killer cell $\left(\mathrm{NK} 1.1^{+}\right)$, eosinophil $\left(\operatorname{Siglec} \mathrm{F}^{+}\right)$, macrophage $\left(\mathrm{F} 4 / 80^{+}\right)$, B cell $\left(\mathrm{B} 220^{+}\right)$, CD4 T cell $\left(\mathrm{CD} 4^{+}\right)$, CD4 T-regulatory cell $\left(\mathrm{CD}^{+} \mathrm{CD}^{2} 5^{+} \mathrm{FoxP}^{+}\right)$, CD8 $\mathrm{T}$ cell $\left(\mathrm{CD} 8^{+}\right)$or $\mathrm{CD} 8 \mathrm{~T}$ suppressor cell $\left(\mathrm{CD}^{+} \mathrm{CD} 103^{+}\right)$frequency or number between $N t n-1^{+/-}$mice and $N t n-1^{+/+}$controls (data not shown). However, following 3 (figure 4A,B) and 7 days (figure 4C) of DSS, a dramatic and sustained increase was observed in the frequency and number of neutrophils $\left(\mathrm{Gr}-1^{+}\right.$cells) in the LP of $N t n-1^{+/-}$mice compared with $N t n-1^{+/+}$. Specificity of the netrin-1 effect on this granulocyte population is demonstrated by the dot plot revealing no change in eosinophil frequency (SiglecF ${ }^{+}$cells) between the two groups (figure 4A). Additionally, expression levels of the pro-inflammatory cytokines TNF $\alpha$ (figure 4D) and IL-1 $\beta$ (figure 4E) were significantly enhanced in $N t n-1^{+/-}$mice following 7 days of DSS, confirming the exaggerated inflammatory response observed in these mice.

\section{Netrin-1 treatment attenuates disease severity during experimental colitis}

The observation that netrin-1 deficiency exacerbates DSS-colitis directed us to investigate the consequences of supplementation with netrin-1 in this model. Mice that were treated with recombinant mouse netrin- 1 for 7 days $(1 \mu \mathrm{g} / \mathrm{mouse} /$ day $)$ experienced diminished disease severity as demonstrated by body weight loss, disease activity index, histologic damage and colonic shortening (figure $5 \mathrm{~A}-\mathrm{D}$, respectively) compared with vehicle-treated controls. Reduced inflammation in these mice was indicated by decreased TNF $\alpha$ and IL-1 $\beta$ colonic expression following netrin-1 treatment (figure $5 \mathrm{E}-\mathrm{F}$ ). These observations indicate that treatment with exogenous netrin-1 exerts a protective effect in experimental colitis.

\section{Netrin-1 does not affect epithelial barrier function}

Epithelial barrier dysfunction is a key feature of human UC and a major component of DSS-colitis. ${ }^{32}$ The prominent influx of neutrophils into the LP of $N t n-1^{+/-}$mice compared to wild-type controls $\left(N t n-1^{+/+}\right)$, particularly at early onset of DSS-colitis (day 3, figure 4B), led us to investigate netrin-1 effects on epithelial barrier function. Furthermore, netrin-1 signalling has been implicated in antiapoptotic effects on intestinal epithelial cells $^{33}$; therefore, we could hypothesise that netrin- 1 mediates protection during DSS by inhibiting intestinal epithelial cell apoptosis.

For this purpose, serum levels of FITC were determined following gavage with FITC-dextran $(4 \mathrm{kDa})$ in $N t n-1^{+/-}$mice compared to wild-type controls after 3 days of DSS. This day was chosen as epithelial barrier dysfunction occurs at early time points post $\mathrm{DSS}^{32}$ and $N t_{n-1}{ }^{+/-}$mice experience significantly differential body weight loss by day 3 (figure $3 \mathrm{~A}$ ). No difference in epithelial permeability was noted between the $\mathrm{Ntn}_{-1}{ }^{+/-}$and wild-type water controls. DSS exposure resulted in a significant increase in serum FITC in wild-type mice compared to their water controls. Importantly, we observed no additional increase in permeability in $\mathrm{Ntn-1^{+/ }}$ mice following DSS compared to wild-type counterparts (figure 6A). This indicates that loss of netrin-1 expression does not directly affect epithelial barrier function. This study was repeated in mice treated with exogenous netrin-1 ( $1 \mu \mathrm{g} / \mathrm{mouse} /$ day $)$ at the same time point 
Figure 3 Disease activity in mice with partial netrin-1 deficiency during DSS-colitis. Gender-, age- and weightmatched mice with partial netrin-1 deficiency (Ntn-1 ${ }^{+/-}$) and their wildtype controls (Ntn-1 $1^{+/+}$) were exposed to DSS $(4.5 \%)$ for 7 days, followed by sacrifice and harvesting of the whole colon by blunt dissection. (A) Daily weight measurements were obtained for each group of mice. $p<0.05$ as measured by analysis of variance (ANOVA). (B) Daily disease activity measurements encompassing weight, stool consistency and presence of blood were assessed for each group of mice. $\mathrm{p}<0.05$ as measured by ANOVA. (C) At harvest, colon length was measured for each mouse and is displayed as the mean \pm SEM. (D) Representative histological sections from whole colon of Ntn-1 ${ }^{+/+}$or $\mathrm{Ntn}-1^{+/-}$mice harvested following 7 days of DSS or water. Bar represents $100 \mu \mathrm{m}$. Images acquired at 10X using Olympus BX51. (E) Blinded histological scoring of colonic tissue post DSS. All results are representative of three independent experiments with six to eight mice per group and are displayed as mean \pm SEM.
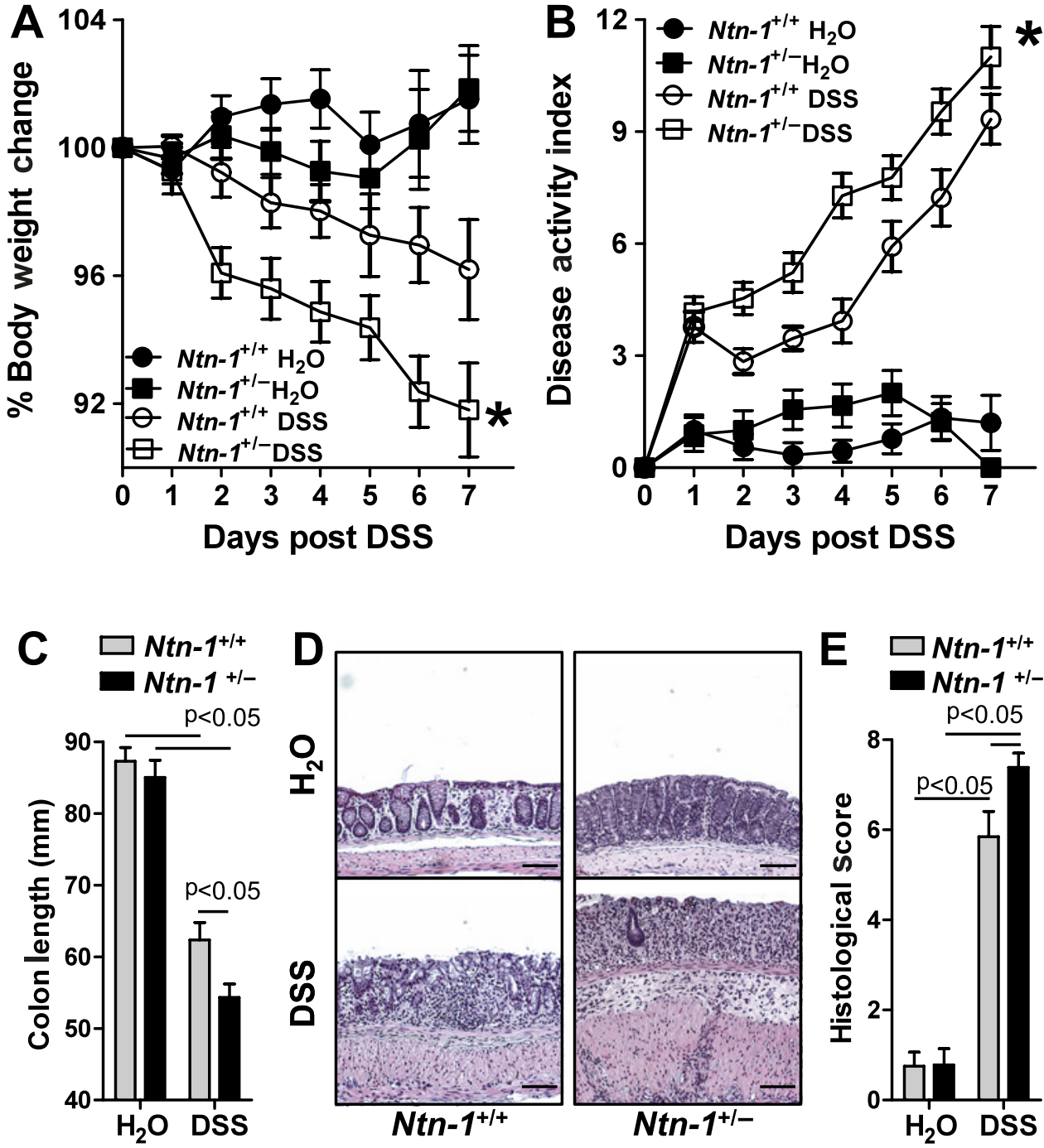

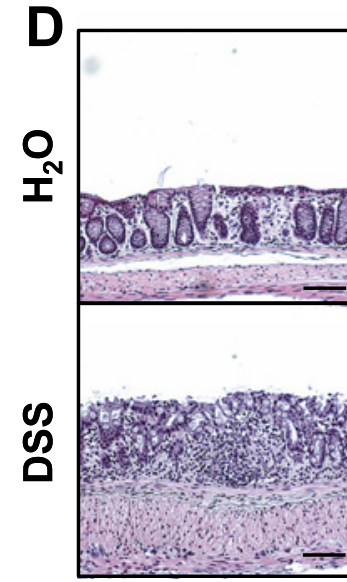

Ntn-1+/+

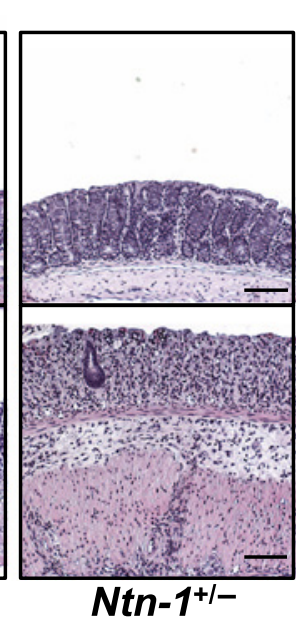

post DSS. These mice experience improved disease outcome compared to vehicle-treated controls (figure 5). Mice exposed to DSS demonstrated significant increase in serum FITC compared to water controls as previously noted ${ }^{32}$; netrin-1 treatment had no effect on barrier function as measured by serum FITC levels (figure 6B). To more specifically analyse the effect of netrin-1 on intestinal epithelial cell apoptosis, colonic sections from mice treated with vehicle or netrin-1 exposed to water or DSS for 6 days were TUNEL stained (figure $6 \mathrm{C}, \mathrm{D}$ ). Limited basal intestinal epithelial cell apoptosis was identified in water controls which was unaltered by netrin-1 treatment (figure 6C,D; arrows indicate apoptotic nuclei). The percentage of apoptotic colonic epithelial cells was dramatically enhanced in mice exposed to DSS, as previously noted. ${ }^{34}$ Netrin-1 treatment during DSS did not alter the percentage of apoptotic epithelial cells (figure 6C,D). Taken together, these studies indicate that the protection conveyed by endogenous or exogenous netrin-1 during experimental colitis does not primarily involve modulation of intestinal barrier function or epithelial cell apoptosis.

In vitro experiments using Caco-2 intestinal epithelial cells as a model of epithelial barrier function confirmed in vivo findings. High-resistance Caco-2 monolayers were treated with DSS (4.5\%; basolaterally and apically) for $6 \mathrm{~h}$ (figure $6 \mathrm{E}$ ) or cytokine cocktail for $48 \mathrm{~h}$ (basolateral IFN $\gamma$, TNF $\alpha$, IL-1 $\beta$; all at $10 \mathrm{ng} / \mathrm{ml}$; figure $6 \mathrm{~F}, \mathrm{G})$. Co-incubation with netrin-1 at the basolateral membrane or at both sides of the membrane (data not shown for cytomix) had no effect on barrier function as measured by transepithelial electrical resistance (TEER; figure $6 \mathrm{E}, \mathrm{F}$ ) or by FITCdextran $(3 \mathrm{kDa})$ flux performed at $48 \mathrm{~h}$ post cytomix treatment (figure $6 \mathrm{G}$ ), supporting in vivo findings that netrin-1 does not directly modulate the intestinal epithelial barrier function.

\section{Netrin-1 limits colonic leucocyte infiltration to attenuate inflammation in experimental colitis}

Our previous observation of increased neutrophil $\left(\mathrm{Gr}-1^{+}\right.$cells) accumulation within the LP of netrin-1-insufficient mice (figure $4 \mathrm{~A}-\mathrm{D})$ coupled with studies indicating that netrin-1 regulates leucocyte transmigration ${ }^{2} 6$ prompted investigation of the effects of exogenous netrin-1 on leucocyte migration. Transmigration of neutrophils across the epithelial barrier is a morphologic hallmark of colitis. ${ }^{29} 3536$ Initial investigation revealed that netrin-1 suppressed chemo-attractant-induced (Formyl-Methinyl-Leucyl-Phenylalanine; fMLP) human neutrophil (PMN) transmigration across a Caco-2 intestinal epithelial barrier in a concentration-dependent manner (figure 7A), in support of previous observations ${ }^{26}$ and our conclusions from initial in vivo studies (figure 4A-C). Flow cytometric analysis revealed that the frequency and number of $\mathrm{Gr}-1^{+}$neutrophils within the LP were significantly attenuated following netrin-1 treatment during DSS compared to vehicle-treated controls (figure 7B). Previous studies demonstrated that netrin-1 can 

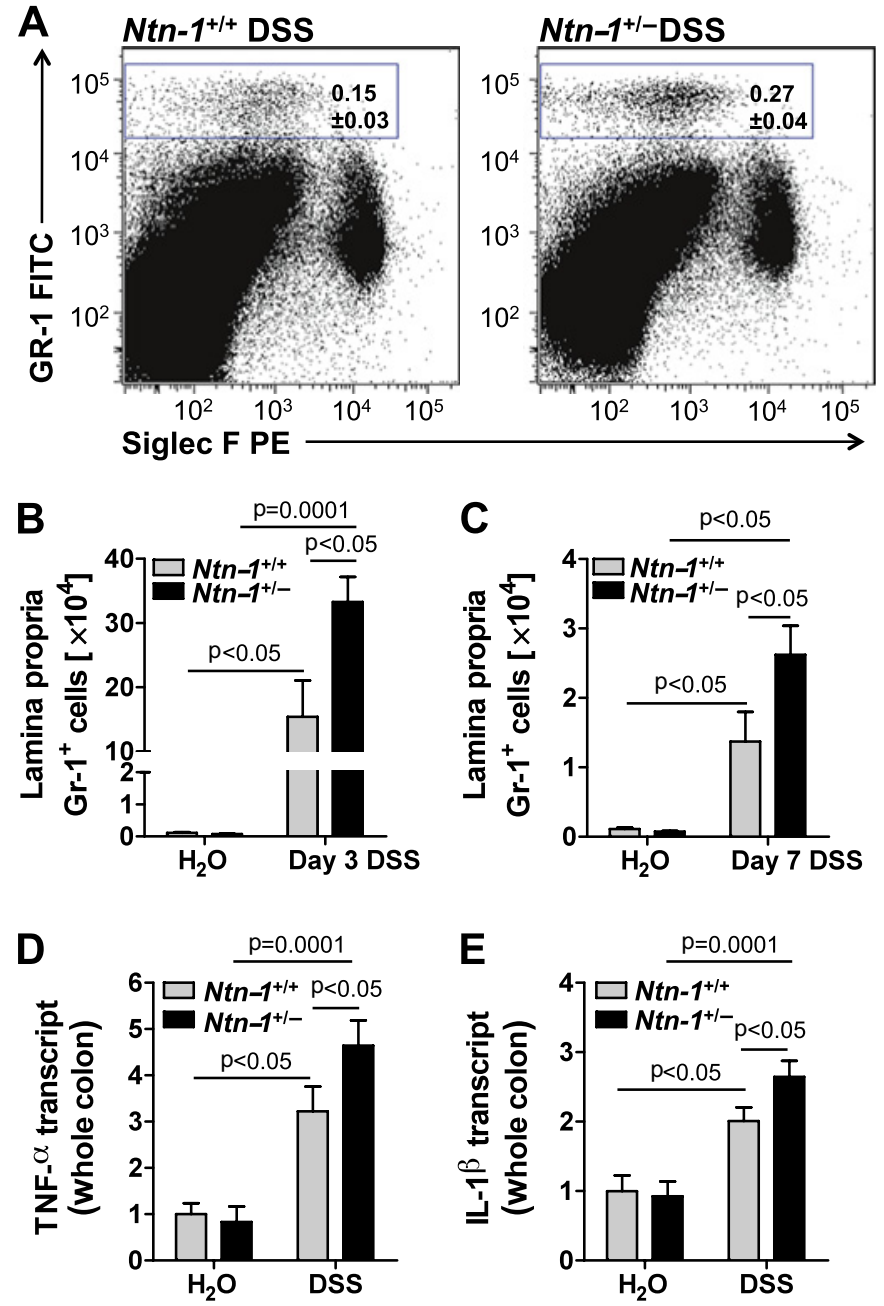

Figure 4 Colonic inflammatory infiltrate following DSS-colitis in Ntn- $1^{+/}$ + and Ntn- ${ }^{+/-}$mice. Gender-, age- and weight-matched mice with partial netrin-1 deficiency (Ntn-1 ${ }^{+-}$) and their wild-type controls (Ntn$1^{+/+}$) were exposed to DSS (4.5\%) for 3 or 7 days, followed by sacrifice and harvesting of the whole colon by blunt dissection. Colonic lamina propria leucocytes were isolated, and flow cytometric analysis of GR-1 ${ }^{+}$ (neutrophils) and $\mathrm{SiglecF}^{+}$(eosinophils) granulocytes was performed. (A) Representative dot plots showing GR-1 $1^{+}$granulocytes from the colonic lamina propria of $\mathrm{Ntn-1^{+/+ }}$ and $\mathrm{Ntn-1} \mathrm{1}^{+/-}$mice post DSS. (B) The number of $\mathrm{GR}-1^{+}$granulocytes in the colonic lamina propria following 3 days of water or DSS in $\mathrm{Ntn}-\mathrm{1}^{+/+}$and $\mathrm{Ntn-1} \mathrm{1}^{+/-}$mice. (C) The number of GR-1 ${ }^{+}$ granulocytes in the colonic lamina propria following 7 days of water or DSS in Ntn- $1^{+/+}$and $N t n-1^{+/-}$mice. (D, E) Following whole colon harvest, total RNA was extracted, and tumor necrosis factor (TNF) $\alpha$ (D) or interleukin (IL) $1 \beta$ (E) transcript levels were determined by real-time reverse transcriptase polymerase chain reaction (RT-PCR). Gene expression was calculated relative to $\beta$-actin and expressed as fold change relative to water-exposed $N t n-1^{+/+}$mice \pm SEM. Results represent two independent experiments with four mice per group (flow cytometry) or six to eight mice per group (RT-PCR).

limit neutrophil and monocyte recruitment during instances of acute tissue injury. ${ }^{6} 10$ Given that the anti-Gr-1 antibody recognises Ly-6G and Ly-6C antigens, with Ly-6G representing a specific neutrophil surface marker and Ly-6C acting as a marker for inflammatory monocytes/macrophages, ${ }^{37}$ we sought to dissect whether netrin-1 was limiting transmigration of both cell types to the LP during DSS-colitis. Flow cytometric analysis of leucocytes isolated from the LP of vehicle- and netrin-1treated mice demonstrated that the frequency and number of Ly- $6 \mathrm{G}^{\mathrm{Hi}} \mathrm{CD} 11 \mathrm{~b}^{+}$neutrophils were considerably increased during
DSS-colitis compared to water controls, with both parameters being significantly diminished by netrin-1 treatment (supplementary figure $1 \mathrm{~A}-\mathrm{C}$ ). To negate neutrophilic contribution due to binding of Ly-6C to neutrophils, the frequency of monocyte populations was assessed gating on Ly-6G negative cells. The frequency and number of Ly-6C high (Ly6G $-\mathrm{Cy}-6 \mathrm{C}^{\mathrm{Hi}} \mathrm{CD} 11 \mathrm{~b}^{+}$) and Ly-6C intermediate (Ly6G $\mathrm{C}^{-} \mathrm{Ly}-6 \mathrm{C}^{\text {Int }} \mathrm{CD} 11 \mathrm{~b}^{+}$) monocytes/ macrophages were augmented during DSS but were not significantly altered by netrin-1 (supplementary figure $1 \mathrm{D}, \mathrm{E})$. Therefore, detailed flow cytometric analysis using two distinct markers for neutrophil recognition demonstrates that netrin-1 can significantly attenuate neutrophil recruitment to the LP during DSS. Our analysis reveals no distinct effect of netrin-1 on monocyte/macrophage tissue infiltration during DSS. Therefore, we conclude that netrin-1 is part of an endogenous protective pathway within the colonic epithelium that dampens DSS-colitis through preferential limitation of neutrophil tissue infiltration.

\section{Purinergic signalling pathways contribute to netrin-1-mediated protection from experimental colitis}

Having identified that netrin-1 mediates protection during DSScolitis through limitation of leucocyte recruitment, we proceeded to investigate the specific netrin-1 receptor involved. Previous study has demonstrated significant levels of expression of the UNC5B (uncoordinated receptor 5B) receptor and Adora $2 b$ (A2B adenosine receptor) on leucocytes which have each been demonstrated to mediate netrin-1 protective effects in models of acute inflammation. ${ }^{2} 6810$ Taking this into account, we initially performed an antibody blocking study of the UNC5B receptor using a strategy shown to be effective in reversing netrin-1-mediated protection in a model of renal ischaemia. ${ }^{10}$ Anti-UNC5B blocking antibody or control immunoglobulin (Ig) G was administered ( $800 \mu \mathrm{g} / \mathrm{kg} / \mathrm{mouse}) 1$ day prior to commencement of netrin-1 delivery $(1 \mu \mathrm{g} / \mathrm{mouse} /$ day $)$ and 2 days prior to DSS with blocking antibody or control IgG administration at 2-day intervals until day 6 post DSS (day -2 , $0,2,4)$ when mice were sacrificed. Consistent with previous results (figure 5), netrin-1 significantly attenuated severity of DSS-colitis as measured by weight loss, colon shortening and histologic tissue injury (figure $8 \mathrm{~A}-\mathrm{C}$, respectively). Co-administration of a UNC5B blocking antibody did not alter netrin-1mediated protection, with mice exhibiting no differences in weight loss, colon length or histologic injury to those treated with control IgG (figure $8 \mathrm{~A}-\mathrm{C}$ ), thus indicating that netrin-1 was not acting through UNC5B to protect in DSS.

Subsequent studies were performed with Adora2b-deficient mice (Adora2b $\mathrm{b}^{-1-}$; figure $8 \mathrm{D}-\mathrm{F}$ ). Adora $2 \mathrm{~b}^{-1-}$ mice treated with netrin-1 ( $1 \mu \mathrm{g} / \mathrm{mouse} /$ day with subcutaneous pump) demonstrated comparable disease activity as measured by weight loss, colon shortening or histologic injury (figure 8D-F) compared to vehicle-treated Adora $2 b^{-/-}$controls. Consistent with previous studies in acute inflammatory models, ${ }^{2}{ }^{8}$ we observed that netrin-1-mediated protection during DSS-colitis involves adenosine receptors.

\section{DISCUSSION}

CD and UC are chronic relapsing disorders of the gastrointestinal tract which exert devastating personal and professional consequences on the approximately 1 million sufferers. ${ }^{14}$ Disease progression is relentless, with the eventual outcome being surgical intervention for up to $70 \%$ of patients. ${ }^{38} 39$ The era of the biologics has ushered in hope for improving therapeutic strategies; however, this approach is not without serious 
Figure 5 Exogenous netrin-1 treatment in DSS-colitis. Gender-, ageand weight-matched $\mathrm{C} 57 \mathrm{BL} / 6$ mice were treated with netrin-1 $(1 \mu \mathrm{g} /$ mouse/day) or vehicle ( $1 \%$ bovine serum albumin (BSA)/phosphatebuffered saline (PBS)) using a subcutaneous osmotic pump beginning 1 day prior to administration of (day -1 ) water or DSS (4.5\%) for 6 days, followed by sacrifice and harvesting of the whole colon by blunt dissection. (A) Daily weight measurements were obtained for each group of mice. $p<0.05$ by analysis of variance (ANOVA). (B) Assessment of daily disease activity measurements, encompassing weight, stool consistency and presence of blood, was performed for each group of mice. $\mathrm{p}<0.05$ by ANOVA. (C) Representative histological images from vehicle- and netrin-1-treated mice exposed to water or $4.5 \%$ DSS. Bar represents $100 \mu \mathrm{m}$. Images acquired at 10X using Olympus BX51. Bar graph of blinded histological scoring of colonic tissue post treatment displayed as the mean \pm SEM. (D) Colon length measurements for each mouse were taken following harvest at day 6 post DSS and are displayed as mean \pm SEM. $(E, F)$ Following whole colon harvest, total RNA was extracted, and tumor necrosis factor (TNF) $\alpha$ (E) or interleukin (IL) $1 \beta(\mathrm{F})$ transcript levels were determined by real-time reverse transcriptase polymerase chain reaction (RT-PCR). Gene expression was calculated relative to $\beta$-actin and expressed as fold change relative to water-exposed vehicle-treated mice \pm SEM. Results represent 3 eight mice per group. independent experiments with six to
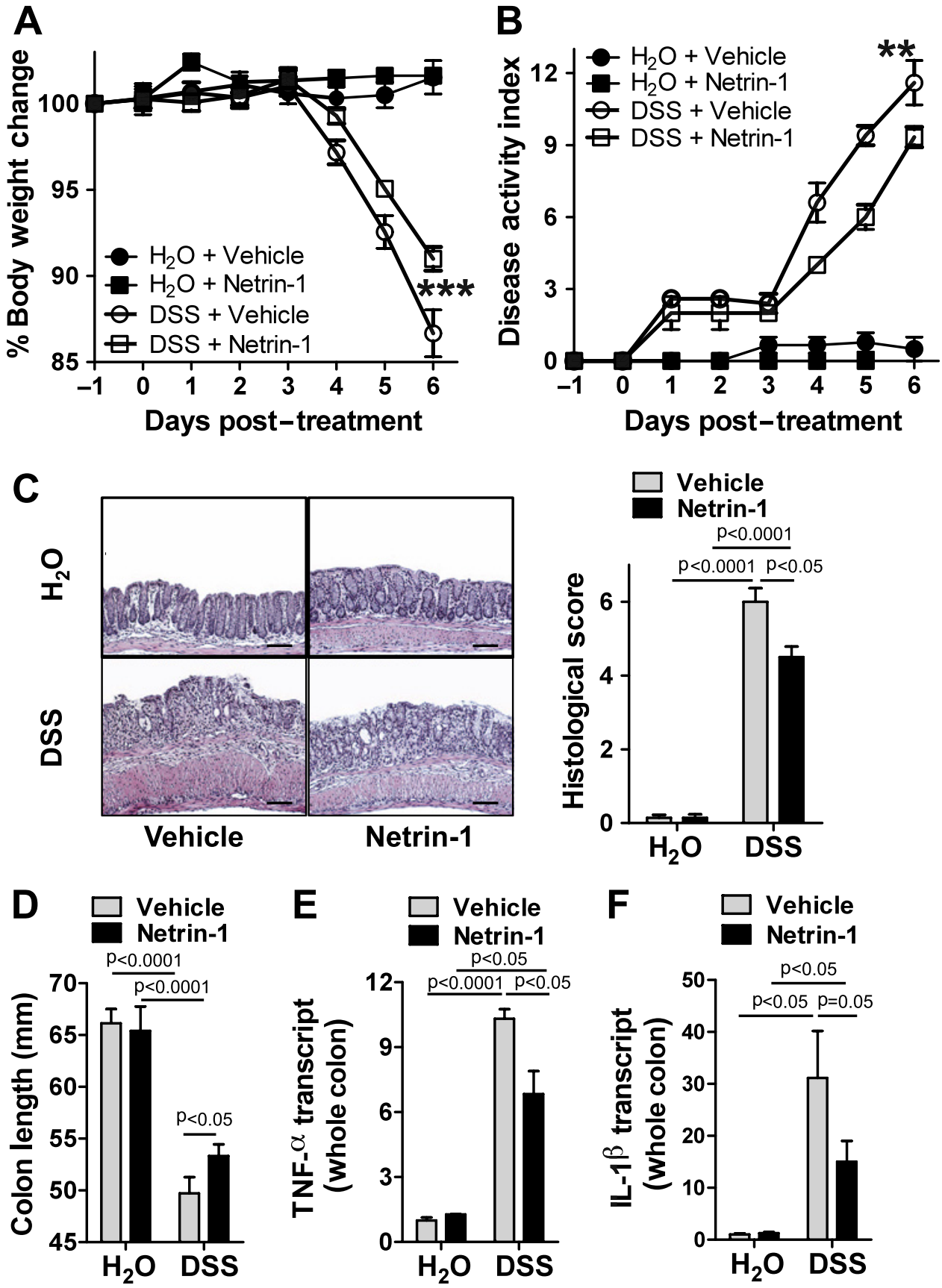

contraindications and adverse events. ${ }^{14}$ There remains an urgent need for novel therapeutics particularly to control the exaggerated inflammation within the intestine of patients with IBD. Recent studies have implicated the neuronal guidance molecule netrin-1 in mediating tissue inflammatory responses. ${ }^{2}$ 6-9 Particularly, netrin-1 expression was shown to be induced under conditions of limited oxygen availability and to ameliorate hypoxia-driven inflammation. ${ }^{2}$ Based on these findings, we hypothesised that netrin-1 could represent an endogenous anti-inflammatory in the context of IBD-a condition that is also characterised by profound tissue hypoxia. ${ }^{19}$ Excitingly, our studies revealed that mice with partial netrin-1 deficiency (Ntn$1^{+/-}$) demonstrated increased disease susceptibility in a model of DSS-colitis. This was associated with a robust neutrophil infiltration into the colonic LP and a significant increase in tissue cytokine levels in the $\mathrm{Ntn-1^{+/- }}$ mice compared to their wild-type controls. Conversely, supplementation with exogenous netrin-1 was associated with attenuated weight loss, improved tissue histology and diminished colonic inflammation. Moreover, mechanistic studies pointed towards a role for netrin- 1 in preferentially dampening neutrophil tissue infiltration rather than improving intestinal barrier function or attenuating intestinal epithelial cell apoptosis. Studies using antibody blockade of the UNC5B receptor or gene-targeted mice for the Adora2b point towards adenosine-receptor-mediated netrin-1 protection during DSS-colitis. Together, these studies demonstrate for the first time a role of netrin-1 in the suppression of intestinal inflammation as occurs in the context of IBD.

Consistent with our observations, previous studies have demonstrated that netrin-1 is induced by inflammatory signalling pathways in intestinal epithelia via NFKB (nuclear factor $\kappa \mathrm{B}$ ) signalling. ${ }^{27}$ We demonstrate that netrin-1 expression is enhanced at the protein and mRNA level during the course of DSS-colitis. Despite netrin-1 mRNA upregulation by inflammatory 
Figure 6 Netrin-1 effects on the intestinal epithelial barrier. (A) Mice with partial netrin-1 deficiency $\left(N t n-1^{+/}\right)$and their wild-type controls $\left(\mathrm{Ntn}-1^{+/+}\right)$were exposed to DSS for 3 days (described in figure 3 ) prior to oral gavage with FITC-dextran $(4 \mathrm{kDa}$; $0.6 \mathrm{mg} / \mathrm{g}$ at $80 \mathrm{mg} / \mathrm{ml}$ ). Fluorescence measurement $(478 \mathrm{~nm})$ was used to determine FITC in the serum $4 \mathrm{~h}$ later and is displayed as mean FITC $(\mu \mathrm{g} /$ $\mathrm{ml}) \pm$ SEM. (B) Mice with vehicle or netrin-1 treatment (described in figure 5) were exposed to DSS for 3 days prior to oral gavage with FITC-dextran (4 kDa $0.6 \mathrm{mg} / \mathrm{g}$ at $80 \mathrm{mg} / \mathrm{ml}$ ), and serum FITC levels were determined and displayed as described in $A$.

(C) Representative images of apoptotic colonic epithelial cells identified by TUNEL staining of paraffin-embedded colonic sections following treatment of mice with netrin-1 or vehicle and exposure to DSS for 6 days (described in figure 5). Images were acquired at 20X using a Nikon Eclipse Ti-S microscope, DS-Fi1 1.0X camera and represent at least six mice per group. (D) Quantification of the percentage of apoptotic colonic epithelial cells in vehicle- or netrin-1-treated mice following 6 days of DSS exposure (described in figure 5). Scoring was carried out in a blinded fashion with counting of 600 cells over three randomly selected fields per section with six mice per group. (E) Caco-2 intestinal epithelial monolayers cultured as monolayers on transwell permeable supports for at least 3 weeks prior were exposed to $4.5 \%$ DSS in the apical and basolateral chamber, with vehicle or human recombinant netrin-1 $(500 \mathrm{ng} /$ ml) co-treatment. Transepithelial electrical resistance (TEER) measurements were taken at time intervals indicated ( $24 \mathrm{~h}$ not shown) and are displayed as mean resistance measurements $\left(0 \mathrm{hms} . \mathrm{cm}^{2}\right) \pm$ SEM. (F) Caco-2 cells were cultured as described (E) prior to basolateral treatment with tumor necrosis factor (TNF) $\alpha$, interleukin (IL) $1 \beta$ and interferon (IFN) $\gamma$ (cytomix; all $10 \mathrm{ng} / \mathrm{ml}$ ) plus vehicle or human recombinant netrin-1 $(500 \mathrm{ng} / \mathrm{ml})$ for $48 \mathrm{~h}$. TEER measurements were taken at time intervals indicated. (G) FITC-dextran (3 kDa; $250 \mu \mathrm{g} / \mathrm{ml})$ was applied to the apical compartment of Caco-2 monolayers treated for $48 \mathrm{~h}$ as described in F. Appearance of FITC in the basolateral compartment was measured over a $3 \mathrm{~h}$ period and analysed as described in A. Results displayed as the apparent permeability of the monolayer (Papp; $\mathrm{cm}^{2} / \mathrm{s}$ ). In vivo FITC measurements represent five mice per group. In vitro experiments represent four individual wells per treatment from two separate experiments.

stimulation of HMEC-1 endothelial cells, immunohistochemical analysis of the murine colon during DSS revealed limited, if any, detectable expression of netrin-1 at the level of the endothelium. However, netrin-1 protein was robustly expressed in the intestinal epithelium where it appears to be increased during DSS exposure. This correlates with enhanced netrin-1 expression observed in the intestinal epithelia of patients with $\mathrm{CD}$ and UC. ${ }^{15}$ Consistent with these studies, netrin- 1 induction in IBD could involve the NFKB pathway. ${ }^{27}$ However, a recent study demonstrates that HIF-1 $\alpha$ (hypoxia-inducible factor $1 \alpha$ ), a transcription factor stabilised in intestinal epithelia in murine models of IBD, ${ }^{19}$ can induce netrin-1 expression in intestinal epithelia. ${ }^{2}$ Additionally, the Ets-1 transcription factor that is upregulated during $\mathrm{IBD}^{40}$ has been shown to induce netrin- 1 expression in melanocytes. ${ }^{41}$ Therefore, multiple pathways can regulate netrin1 expression in the context of inflammation.

Immunohistochemical analysis revealed consistent expression of netrin-1 in colonic myenteric neural units during DSS. Recent studies demonstrated a role for netrin-1 in the developing enteric nervous system where enteric neural-derived crest cells produce netrin-1. ${ }^{42}$ This intriguing observation led us to analyse the enteric neuronal anatomy of netrin-1-insufficient mice during DSS-colitis. Independent analysis revealed no difference in the enteric neuronal anatomy of netrin-1-insufficient mice in comparison with their wild-type controls either at baseline or during disease course. We conclude that netrin-1 insufficiency does not alter the neuronal anatomy in adult mice prior to or during DSS. 
A
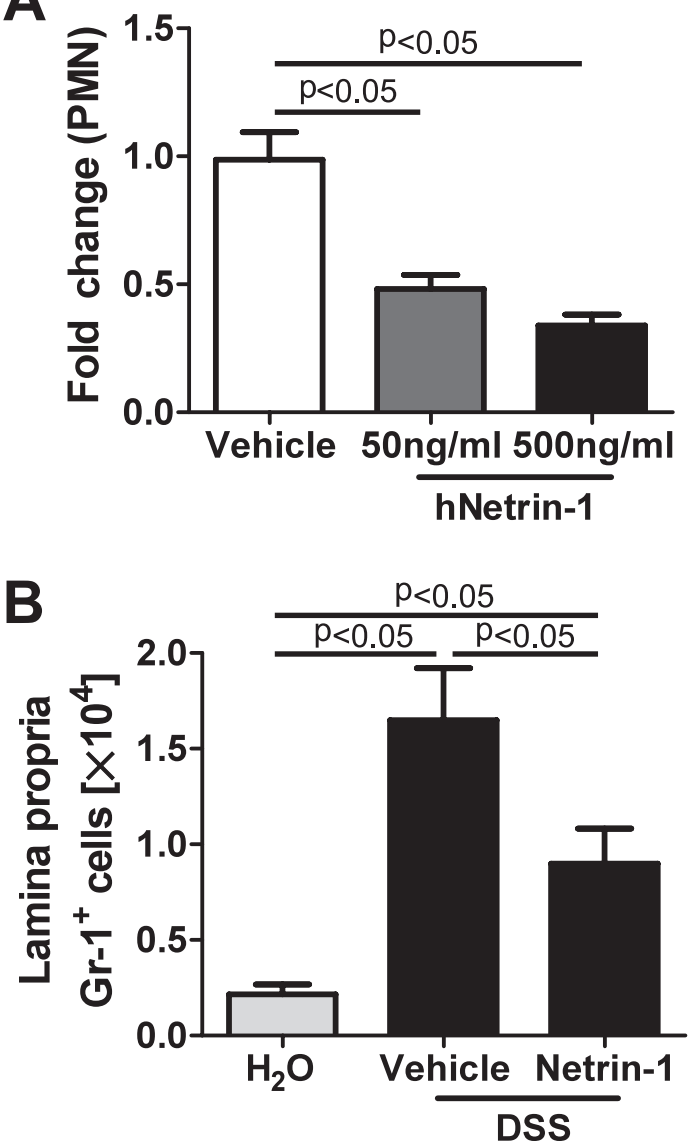

Figure 7 Netrin-1 effects on inflammatory infiltrate in vitro and in vivo. (A) Neutrophils (PMN) were harvested from whole human blood and applied at $1 \times 10^{6}$ PMN in the presence of vehicle or increasing concentrations of human recombinant netrin- $1(50-500 \mathrm{ng} / \mathrm{ml})$ to the basolateral aspect of Caco-2 intestinal epithelial cells grown to confluence on inverted permeable supports. fMLP $(1 \mu \mathrm{M})$ was added to the apical aspect of each well. Presence of transmigrated neutrophils in the apical compartment was measured by myeloperoxidase levels and is displayed as the mean fold change in PMN numbers relative to vehicle \pm SEM. Results represent at least triplicate wells from three independent experiments. (B) $\mathrm{C} 57 \mathrm{BL} / 6$ mice were treated with netrin-1 (1 $\mu \mathrm{g} / \mathrm{mouse} /$ day) or vehicle prior to exposure to water or DSS as described in figure 5. Following colon harvest at day 6 post DSS, colonic lamina propria leucocytes were isolated, and flow cytometric analysis of GR-1 ${ }^{+}$(neutrophils) and SiglecF ${ }^{+}$(eosinophils) granulocytes was performed. The number of GR-1 ${ }^{+}$granulocytes is displayed as mean $\pm S E M$ for four mice.

Supporting our observations, an anti-inflammatory effect of netrin-1 has been observed in models of acute inflammation. ${ }^{26-9}$ Originally, netrin-1 was demonstrated to attenuate LPS-induced sepsis through inhibition of leucocyte migration. ${ }^{6}$ Studies in kidney ischaemia-reperfusion injury, ${ }^{7}{ }^{10}$ hypoxia-induced inflammation, ${ }^{2}$ acute lung injury ${ }^{8}{ }^{43}$ and peritonitis ${ }^{9}$ support this observation by demonstrating a tissue-protective role for netrin-1 through suppression of inflammation. Presently, a number of downstream receptor signalling pathways have been implicated in netrin-1-mediated tissue protection observed in these studies. The transmembrane receptors, deleted in colorectal cancer, uncoordinated receptor 5 (mouse UNC5A-D; human UNC5H1-4) and the $\mathrm{A} 2 \mathrm{~B}$ adenosine receptor (ADORA2B/Adora2b) represent the known netrin-1 receptors. ${ }^{2} 68-104445$ Independent studies have demonstrated that either UNC5B or Adora2b expression on leucocytes is responsible for netrin-1 inhibition of tissue inflammation. ${ }^{2}{ }^{68-10}$ Of additional relevance to the present study is the observation that netrin-1 receptors can mediate the apoptotic response of the intestinal epithelium. ${ }^{33}$ Enforced expression of netrin- 1 in the intestinal epithelium resulted in decreased epithelial apoptosis and a modest increase in tumour formation in the presence of APC mutation. ${ }^{33}$ Furthermore, blockade of netrin-1 interaction with epithelial deleted in colorectal cancer receptor enhanced tumour cell death in a model of colorectal cancer progression. ${ }^{15}$ Intestinal epithelial cell apoptosis, along with alterations in epithelial tight junction formation, is central to the mucosal disruption observed in patients with IBD. ${ }^{46} 47$ Similarly, epithelial cell apoptosis and tight junction breakdown are key features of DSS-colitis, while inhibition of apoptosis in this model is barrier protective. ${ }^{34} 48$ Therefore, it is conceivable that antiapoptotic effects of netrin-1 signalling may be central to its beneficial effects during experimental colitis. In vitro studies of cytokine-induced tight junction rearrangement reveal no effect of netrin-1 on this aspect of epithelial permeability. ${ }^{49}$ Importantly, in vitro and in vivo studies indicate that netrin-1 does not influence DSS-induced epithelial barrier disruption and does not have a direct impact on intestinal epithelial cell apoptosis.

In addition to epithelial barrier breakdown, early and persistent infiltration of neutrophils into the LP forming crypt abscesses is a key feature of human disease and murine colitis. ${ }^{29-32}{ }^{36}$ The full extent of the role that neutrophils play during DSS-colitis remains controversial. Studies using direct depletion of neutrophils with anti-Gr1 prior to DSS reveal modest effects of neutrophil depletion on early disease activity but no significant effect on disease outcome. ${ }^{50}{ }^{51}$ However, the authors propose that macrophages are key regulators of DSScolitis that mediate protective effects through limitation of neutrophil infiltration. ${ }^{50}$ Interestingly, one study identified neutrophil depletion leading to worsening disease during DSScolitis. $^{52}$ Conversely, therapeutic targeting of neutrophil recruitment to the colon in models of colitis using blockade of the neutrophil chemokine receptor (CXCR2) or ligand (CXCL5) results in diminished neutrophil recruitment during DSS and amelioration of disease. ${ }^{53-55}$ Taken together, these studies suggest that while complete blockade of neutrophil recruitment during colitis might not be desirable, limitation of the neutrophil influx into the LP would act to control disease severity. Present in crypt abscesses during human disease are other leucocyte populations including monocytes/macrophages and lymphocytes. Therapeutic strategies in human disease and mouse models to deplete granulocytic and monocytic tissue infiltration have met with some success. ${ }^{56} 57$ Therefore, treatment to attenuate tissue infiltration of granulocytes and monocytes may be of therapeutic benefit. In support of these studies, we demonstrate that netrin-1 directly limits trafficking of neutrophils in vitro and in vivo to ameliorate outcome in DSS-colitis. Further dissection of netrin-1 effects on leucocyte recruitment during DSS-colitis indicates that netrin-1 has no discernible effect on monocyte/macrophage recruitment to the colonic LP. Therefore, this study presents the preferential limitation of neutrophil recruitment by netrin-1 during DSS as a novel therapeutic strategy in an acute model of colitis.

Present findings place netrin- 1 as an endogenous protective mediator in experimental colitis. Interestingly, supplementation with netrin-1 in DSS-colitis where its expression is induced is therapeutically effective. A similar phenomenon is observed in two independent studies where stabilisation of the locally produced HIF- $1 \alpha$ transcription factor during experimental colitis 
Figure 8 Specific blockade of netrin-1 receptors during exogenous netrin-1 treatment in DSS-colitis. (A) Gender-, age- and weight-matched $\mathrm{C} 57 \mathrm{BL} / 6$ mice were treated with anti-UNC5B antibody $(800 \mu \mathrm{g} / \mathrm{kg})$ or control goat immunoglobulin $(\mathrm{lg}) \mathrm{G}(800 \mu \mathrm{g} / \mathrm{kg})$ intraperitoneally 2 days prior to administration of water or DSS (3.5\%) followed by repeated administration of blocking antibody or control IgG at days 0,2 , and 4 of DSS exposure. Netrin-1 (1 $\mu \mathrm{g} / \mathrm{mouse} /$ day) or vehicle ( $1 \%$ bovine serum albumin (BSA)/phosphatebuffered saline (PBS)) delivery using a subcutaneous osmotic pump commenced 1 day prior to administration (day -1 ) of water or DSS for 6 days, followed by sacrifice and harvesting of the whole colon by blunt dissection. (A) Daily weight measurements were obtained for each group of mice. $p<0.05$ by analysis of variance (ANOVA). (B) Colon length measurements for each mouse were taken following harvest at day 6 post DSS and are displayed as mean \pm SEM. (C) Representative histological images from (i) water controls, (ii) DSSexposed mice treated with control lgG and BSA/PBS vehicle, (iii) DSS-exposed mice treated with netrin-1 and control $\lg \mathrm{G}$ and (iv) DSS-exposed mice treated with netrin-1 and anti-UNC5b antibody. Bar represents $100 \mu \mathrm{m}$. Images acquired at 10X using an Olympus BX51. (D-F) Gender-, age- and weightmatched $A 2 B$ receptor-deficient mice $\left(\right.$ Adora2 $\left.\mathrm{b}^{-1-}\right)$ were treated with netrin$1(1 \mu \mathrm{g} / \mathrm{mouse} /$ day) or vehicle ( $1 \%$ BSA/PBS) using a subcutaneous osmotic pump commencing 1 day prior to administration (day -1 ) of water or DSS (3.5\%) for 6 days, followed by sacrifice and harvesting of the whole colon by blunt dissection. (D) Daily weight measurements were obtained for each group of mice. (E) Colon length measurements for each mouse were taken following harvest at day 6 post DSS and are displayed as mean \pm SEM. (F) Representative histological images from (i) water controls, (ii) DSS-exposed mice treated with BSA/PBS vehicle and (iii) DSS-exposed mice treated with netrin-1. Bar represents $100 \mu \mathrm{m}$. Images acquired at 10X using an Olympus BX51. Experiments are representative of six to eight mice per group.

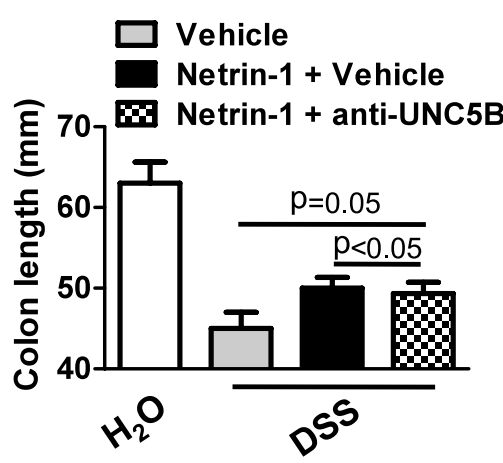

C
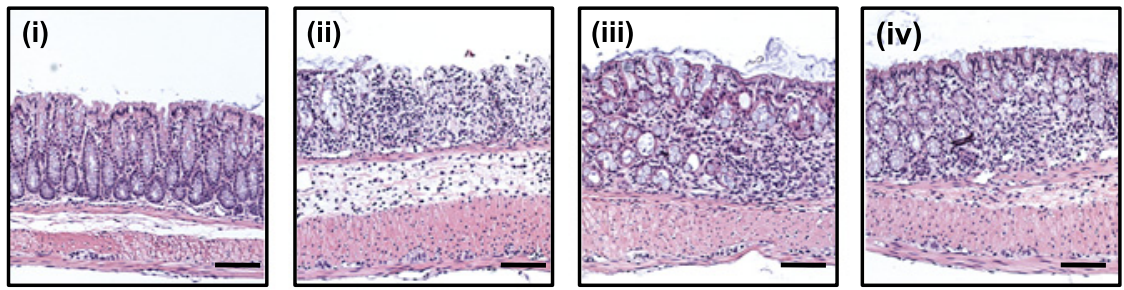

D
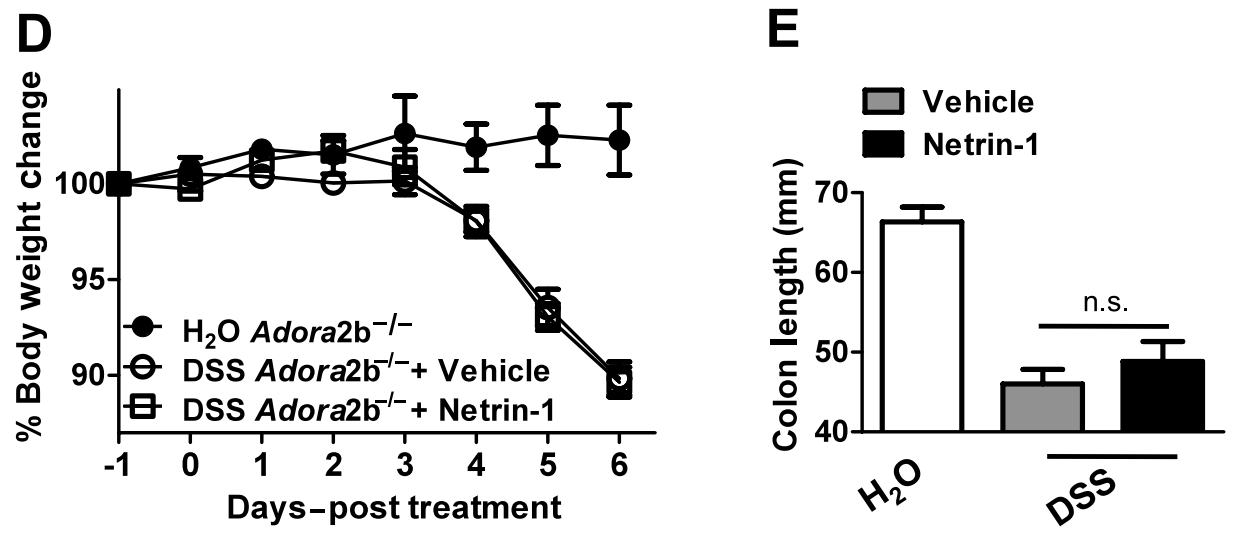

$\mathbf{F}$
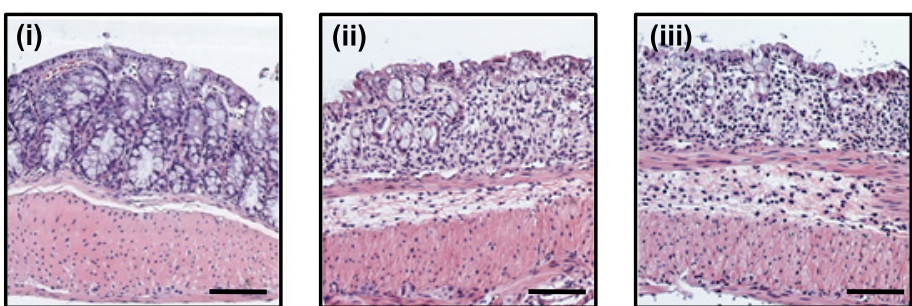

is of therapeutic benefit. ${ }^{16} 18$ These studies support the potential benefit of enhancing in vivo protective responses for therapeutic intervention.

As previously mentioned, netrin-1 has an array of receptors through which it mediates tissue-protective effects. We focused our studies on receptors that have been implicated in mediating netrin-1-driven inhibition of leucocyte recruitment in models of acute inflammation, the UNC5B and the Adora2b. Lack of functional Adora $2 \mathrm{~b}$ abrogated the protective effect of netrin- 1 on DSS-colitis. These studies implicate this purinergic signalling pathway in netrin-1-mediated protection in experimental colitis.

Consideration of netrin-1 as a therapeutic strategy in IBD must take into account the breadth of literature demonstrating the anti-inflammatory component as well as the antiapoptotic role of netrin-1 signalling. Studies indicating the involvement of netrin- 1 in malignant transformation in the intestine, ${ }^{15}$ coupled with our observation that netrin-1 significantly attenuates intestinal leucocyte infiltration in experimental colitis, point to a tailored therapeutic strategy with netrin-1 administration perhaps being more suited to treat periods of disease flare rather than a maintenance treatment.

In summary, our present studies demonstrate for the first time that netrin-1 expression is an endogenous tissue-protective mechanism in experimental colitis. Therapeutic studies indicate that targeting netrin-1 may be useful in treatment of aberrant intestinal leucocyte accumulation, as occurs in colitis. 
Acknowledgements The authors would like to thank Marc Tessier-Lavigne for providing the Ntn-1 mice for this project. Thank you to Sean P. Colgan, Almut Grenz, Eric T. Clambey, Eoin N. McNamee, Kelley Brodsky, Julee Dalton, Timothy Lubbert and Douglas Ridyard for technical assistance as well as manuscript discussion.

Funding The present manuscript is supported by United States National Institutes of Health grant R01-HL0921, R01-DK083385 and R01HL098294 to HKE, and Crohn's and Colitis Foundation Senior Research Award to HKE. Supported by Crohn's and Colitis Foundation Fellowship to CMA, CBC and JCM. Supported in part by the Histology and Imaging Core of NIDCD Grant P30 DC04657 to D. Restrepo and T. Finger

Competing interests None.

Contributors All authors contributed to data generation, data analysis and interpretation as well as writing of the manuscript.

Provenance and peer review Not commissioned; externally peer reviewed.

\section{REFERENCES}

1. Serafini T, Kennedy TE, Galko MJ, et al. The netrins define a family of axon outgrowthpromoting proteins homologous to C. elegans UNC-6. Cell 1994;78:409-24.

2. Rosenberger $\mathbf{P}$, Schwab JM, Mirakaj V, et al. Hypoxia-inducible factor-dependent induction of netrin-1 dampens inflammation caused by hypoxia. Nat Immunol 2009;10:195-202.

3. Yebra M, Montgomery AM, Diaferia GR, et al. Recognition of the neural chemoattractant Netrin-1 by integrins alpha6beta4 and alpha3beta1 regulates epithelial cell adhesion and migration. Dev Cell 2003;5:695-707.

4. Strizzi L, Bianco C, Raafat A, et al. Netrin-1 regulates invasion and migration of mouse mammary epithelial cells overexpressing Cripto-1 in vitro and in vivo. J Cell Sci 2005;118:4633-43.

5. Wang W, Reeves WB, Ramesh G. Netrin-1 increases proliferation and migration of renal proximal tubular epithelial cells via the UNC5B receptor. Am J Physiol Renal Physiol 2009;296:F723-9.

6. Ly NP, Komatsuzaki K, Fraser IP, et al. Netrin-1 inhibits leukocyte migration in vitro and in vivo. Proc Natl Acad Sci U S A 2005;102:14729-34.

7. Wang W, Brian Reeves W, Ramesh G. Netrin-1 and kidney injury. I. Netrin-1 protects against ischemia-reperfusion injury of the kidney. Am J Physiol Renal Physiol 2008;294:F739-47.

8. Mirakaj V, Thix CA, Laucher $S$, et al. Netrin-1 dampens pulmonary inflammation during acute lung injury. Am J Respir Crit Care Med 2010;181:815-24.

9. Mirakaj V, Gatidou D, Potzsch C, et al. Netrin-1 signaling dampens inflammatory peritonitis. J Immunol 2011:186:549-55.

10. Tadagavadi RK, Wang W, Ramesh G. Netrin-1 regulates Th1/Th2/Th17 cytokine production and inflammation through UNC5B receptor and protects kidney against ischemia-reperfusion injury. J Immunol 2010;185:3750-8.

11. Kaser A, Zeissig S, Blumberg RS. Inflammatory bowel disease. Annu Rev Immunol 2010;28:573-621.

12. Abraham C, Cho JH. Inflammatory bowel disease. N Engl J Med 2009;361:2066-78.

13. Xavier RJ, Podolsky DK. Unravelling the pathogenesis of inflammatory bowe disease. Nature 2007; 448:427-34.

14. Clark M, Colombel JF, Feagan BC, et al. American gastroenterological association consensus development conference on the use of biologics in the treatment of inflammatory bowel disease, June 21-23, 2006. Gastroenterology 2007;133:312-39.

15. Paradisi A, Maisse C, Coissieux MM, et al. Netrin-1 up-regulation in inflammatory bowel diseases is required for colorectal cancer progression. Proc Natl Acad Sci U S A 2009; 106:17146-51.

16. Robinson A, Keely S, Karhausen J, et al. Mucosal protection by hypoxia-inducible factor prolyl hydroxylase inhibition. Gastroenterology 2008;134:145-55.

17. Colgan SP, Taylor CT. Hypoxia: an alarm signal during intestinal inflammation. Nat Rev Gastroenterol Hepatol 2010;7:281-7.

18. Cummins EP, Seeballuck F, Keely SJ, et al. The hydroxylase inhibitor dimethyloxalylglycine is protective in a murine model of colitis. Gastroenterology 2008:134:156-65.

19. Karhausen J, Furuta GT, Tomaszewski JE, et al. Epithelial hypoxia-inducible factor-1 is protective in murine experimental colitis. J Clin Invest 2004:114:1098-106.

20. Keely S, Ryan SM, Haddleton DM, et al. Dexamethasone-pDMAEMA polymeric conjugates reduce inflammatory biomarkers in human intestinal epithelial monolayers. J Control Release 2009;135:35-43.

21. Parkos CA, Colgan SP, Bacarra AE, et al. Intestinal epithelia (T84) possess basolateral ligands for CD11b/CD18-mediated neutrophil adherence. Am J Physiol 1995:268:C472-9

22. Ades EW, Candal FJ, Swerlick RA, et al. HMEC-1: establishment of an immortalized human microvascular endothelial cell line. J Invest Dermatol 1992;99:683-90.

23. Smith $\mathbf{P}$, Mangan NE, Walsh $\mathrm{CM}$, et al. Infection with a helminth parasite prevents experimental colitis via a macrophage-mediated mechanism. J Immunol 2007:178:4557-66

24. Siegmund B, Fantuzzi G, Rieder F, et al. Neutralization of interleukin-18 reduces severity in murine colitis and intestinal IFN-gamma and TNF-alpha production. Am J Physiol Regul Integr Comp Physiol 2001:281:R1264-73.

25. Frick JS, MacManus CF, Scully M, et al. Contribution of adenosine A2B receptors to inflammatory parameters of experimental colitis. J Immunol 2009;182:4957-64.
26. Denning TL, Wang YC, Patel SR, et al. Lamina propria macrophages and dendritic cells differentially induce regulatory and interleukin 17-producing T cell responses. Nat Immunol 2007;8:1086-94.

27. Paradisi A, Maisse C, Bernet A, et al. NF-kappaB regulates netrin-1 expression and affects the conditional tumor suppressive activity of the netrin-1 receptors. Gastroenterology 2008;135:1248-57.

28. Kennedy TE, Serafini T, de la Torre JR, et al. Netrins are diffusible chemotropic factors for commissural axons in the embryonic spinal cord. Cell 1994;78:425-35

29. Lampinen M, Sangfelt $P$, Taha $Y$, et al. Accumulation, activation, and survival of neutrophils in ulcerative colitis: regulation by locally produced factors in the colon and impact of steroid treatment. Int J Colorectal Dis 2008;23:939-46.

30. Nikolaus S, Bauditz J, Gionchetti P, et al. Increased secretion of pro-inflammatory cytokines by circulating polymorphonuclear neutrophils and regulation by interleukin 10 during intestinal inflammation. Gut 1998:42:470-6.

31. Kucharzik T, Walsh SV, Chen J, et al. Neutrophil transmigration in inflammatory bowel disease is associated with differential expression of epithelial intercellular junction proteins. Am J Pathol 2001:159:2001-9.

32. Yan Y, Kolachala V, Dalmasso G, et al. Temporal and spatial analysis of clinical and molecular parameters in dextran sodium sulfate induced colitis. PLoS One 2009;4:e6073.

33. Mazelin L, Bernet A, Bonod-Bidaud C, et al. Netrin-1 controls colorectal tumorigenesis by regulating apoptosis. Nature 2004;431:80-4.

34. Tambuwala MM, Cummins EP, Lenihan CR, et al. Loss of prolyl hydroxylase-1 protects against colitis through reduced epithelial cell apoptosis and increased barrie function. Gastroenterology 2010;139:2093-101.

35. Jaye DL, Parkos CA. Neutrophil migration across intestinal epithelium. Ann N Y Acad Sci 2000:915:151-61.

36. Kumar NB, Nostrant TT, Appelman HD. The histopathologic spectrum of acute selflimited colitis (acute infectious-type colitis). Am J Surg Pathol 1982;6:523-9.

37. Lee PY, Kumagai Y, Xu Y, et al. IL-1alpha modulates neutrophil recruitment in chronic inflammation induced by hydrocarbon oil. J Immunol 2011;186:1747-54.

38. Wolters FL, Russel MG, Stockbrugger RW. Systematic review: has disease outcome in Crohn's disease changed during the last four decades? Aliment Pharmacol Ther 2004:20:483-96.

39. Sands BE, Arsenault JE, Rosen MJ, et al. Risk of early surgery for Crohn's disease: implications for early treatment strategies. Am J Gastroenterol 2003;98:2712-18.

40. Jedlicka $\mathbf{P}$, Gutierrez-Hartmann A. Ets transcription factors in intestinal morphogenesis, homeostasis and disease. Histol Histopathol 2008:23:1417-24.

41. Kaufmann S, Kuphal S, Schubert T, et al. Functional implication of Netrin expression in malignant melanoma. Cell Oncol 2009:31:415-22.

42. Ratcliffe EM, Fan L, Mohammed TJ, et al. Enteric neurons synthesize netrins and are essential for the development of the vagal sensory innervation of the fetal gut. Dev Neurobiol 2011;71:362-73.

43. Mutz C, Mirakaj V, Vagts DA, et al. The neuronal guidance protein netrin-1 reduces alveolar inflammation in a porcine model of acute lung injury. Crit Care 2010;14:R189.

44. Dieterich HJ, Weissmuller T, Rosenberger P, et al. Effect of hydroxyethyl starch on vascular leak syndrome and neutrophil accumulation during hypoxia. Crit Care Med 2006; 34:1775-82.

45. Cirulli V, Yebra M. Netrins: beyond the brain. Nat Rev Mol Cell Biol 2007:8:296-306

46. Schulzke JD, Bojarski C, Zeissig S, et al. Disrupted barrier function through epithelia cell apoptosis. Ann N Y Acad Sci 2006;1072:288-99.

47. Schulzke JD, Ploeger $S$, Amasheh $M$, et al. Epithelial tight junctions in intestinal inflammation. Ann N Y Acad Sci 2009:1165:294-300.

48. Araki Y, Mukaisyo $K$, Sugihara $H$, et al. Increased apoptosis and decreased proliferation of colonic epithelium in dextran sulfate sodium-induced colitis in mice. Oncol Rep 2010;24:869-74.

49. Bruewer M, Luegering A, Kucharzik T, et al. Proinflammatory cytokines disrupt epithelial barrier function by apoptosis-independent mechanisms. J Immunol 2003:171:6164-72.

50. Qualls JE, Kaplan AM, van Rooijen N, et al. Suppression of experimental colitis by intestinal mononuclear phagocytes. J Leukoc Biol 2006;80:802-15.

51. Berndt BE, Zhang M, Chen GH, et al. The role of dendritic cells in the development of acute dextran sulfate sodium colitis. J Immunol 2007;179:6255-62.

52. Hans W, Scholmerich J, Gross V, et al. Interleukin-12 induced interferon-gamma increases inflammation in acute dextran sulfate sodium induced colitis in mice. Eur Cytokine Netw 2000;11:67-74

53. Farooq SM, Stillie R, Svensson M, et al. Therapeutic effect of blocking CXCR2 on neutrophil recruitment and dextran sodium sulfate-induced colitis. J Pharmacol Exp Ther 2009:329:123-9.

54. Bento AF, Leite DF, Claudino RF, et al. The selective nonpeptide CXCR2 antagonis SB225002 ameliorates acute experimental colitis in mice. J Leukoc Biol 2008:84:1213-21.

55. Kwon JH, Keates AC, Anton PM, et al. Topical antisense oligonucleotide therapy against LIX, an enterocyte-expressed CXC chemokine, reduces murine colitis. $A m$ Physiol Gastrointest Liver Physiol 2005;289:G1075-83.

56. Hanai $\mathbf{H}$, Watanabe F, Saniabadi AR, et al. Therapeutic efficacy of granulocyte and monocyte adsorption apheresis in severe active ulcerative colitis. Dig Dis Sci 2002:47:2349-53.

57. Tokuyama H, Ueha S, Kurachi M, et al. The simultaneous blockade of chemokine receptors CCR2, CCR5 and CXCR3 by a non-peptide chemokine receptor antagonist protects mice from dextran sodium sulfate-mediated colitis. Int Immunol 2005;17:1023-34. 